\title{
An Analysis of Nonlinear Phenomena in a Thermal Micro-Actuator with a Built-In Thermal Position Sensor
}

\author{
A. Bazaei, Member, IEEE, Y. Zhu, Member, IEEE, S.O.R. Moheimani, Fellow, IEEE, and M.R. \\ Yuce, Senior Member, IEEE
}

\begin{abstract}
An analysis of nonlinear effects associated with a chevron thermal micro actuator with a built in thermal position sensor under static conditions is presented in this paper. The nonlinearities present in both actuator and sensor are studied. The phenomena considered for the sensor include: thermal coupling from actuator to sensor and temperature dependence of electrical resistivity. Those considered for the actuator include: non-uniform spatial distribution of temperature in arms, temperature dependency of thermal expansion coefficient, deviation of arm shape from straight line due to physical constraints, and temperature dependence of electrical resistivity.
\end{abstract}

Index Terms-Nonlinear analysis, Electrothermal position microsensor, Analytical models, Thermoelectric microactuators,

\section{INTRODUCTION}

$\mathrm{H}$ igh precision nanopositioning is a necessity in a number of applications, including scanning tunneling microscopy (STM) [1, 2], atomic force microscopy (AFM) [3], nanometrology [4], biology [5], ultrahigh density probe storage system [6-8], and atom manipulations [9, 10]. Nanopositioning in scanning probe microscopy (SPM) is traditionally performed by scanning stages in the form of tripods [11], tubes $[12,13]$, or flexures [14-16]. These nanopositioners typically have high positioning accuracy with a large dynamic range and a wide bandwidth, enabling fast and robust closed-loop position control [17]. Although macro-scale nanopositioners such as piezoelectric actuators can achieve nanometer-scale positioning resolution and accuracy, they are relatively large, expensive, difficult to integrate in MEMS devices, and require high voltage values [18, 19]. Microelectromechanical System (MEMS) nanopositioners have attracted increasing interest recently due to their small size, low cost, fast dynamics and the

A. Bazaei and S.O.R. Moheimani are with School of Electrical Engineering and Computer Science, University of Newcastle Australia, University Drive, Callaghan, NSW 2308, Australia, (corresponding author Ali Bazaei: e-mail: Ali.Bazaei@newcastle.edu.au).

Y. Zhu is with Griffith School of Engineering, Gold Coast Campus, Griffith University, QLD 4222, Australia, e-mail: y.zhu@ griffith.edu.au.

M.R. Yuce is with Department of Electrical and Computer Systems Engineering, Monash University, Clayton, Victoria, Australia. E-mail: Mehmet.Yuce@monash.edu. emergence of applications such as probe-based data storage $[20,21]$. Electrothermal MEMS actuators can provide large forces at moderate voltage values. Hence, they have been used in many applications, such as investigating live biological cells [22] and linear and rotary micromotors [23-26].

Recently, incorporation of a thermal sensing scheme was reported in a MEMS electrostatic actuator [27] and a probebased storage device [28, 29]. Micro-heaters were used to measure the motion of a MEMS micro-scanner with resolution of less than $1 \mathrm{~nm}$. Also, the positions of electrothermal actuators were precisely controlled in feedback loops by onchip sensors that work based on piezoresistivity [30] or thermal variation of electrical resistivity [31]. Compared to the capacitive schemes, thermal actuation and sensing involve lower actuation voltages, smaller footprint, and less complicated circuitry and integration mechanisms [32].

Efficient design of micro actuators and sensors in MEMS and NEMS devices can be significantly improved if reliable models are provided in advance. As electrothermal sensing in MEMS has been under investigation for only a few years, few modeling work can be found in the literature. A single beam electrothermal sensor was modeled in [33]. However, There is a need to develop a better understanding of the thermal coupling from actuator to sensor and the differential sensing scheme used in the on-chip sensing [31]. MEMS-based electrothermal actuation has been studied extensively [34, 35]. However, in most cases, small strains and displacement are considered and the shear deformation of the beams is assumed to be negligible. These assumptions are not applicable in MEMS devices that undergo large displacements and are thus nonlinear, e.g. nanopositioning stages with large dynamic ranges.

This work considers modeling of various nonlinear effects in a chevron thermal micro-actuator with a built in thermal sensor. The nonlinearities are included in a step-by-step and cumulative manner, such that the effects of all nonlinear phenomena are considered simultaneously at last. The objective of this paper is to develop simple analytical methods that include major nonlinear phenomena affecting the actuator and sensor under static conditions. Simulations are validated by experimental results obtained from a MEMS nanopositioner equipped with electrothermal actuation and sensing. 


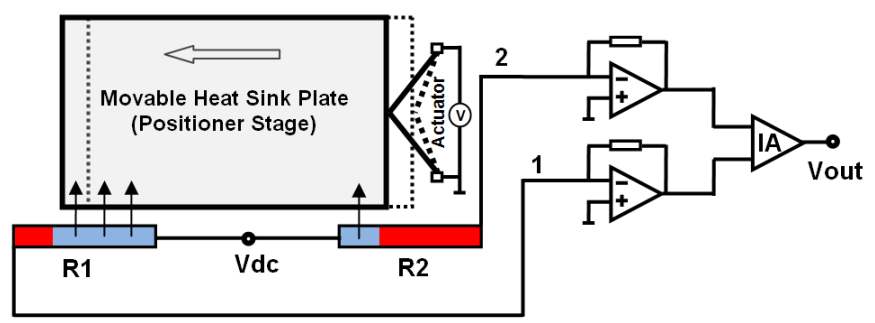

Fig. 1. Schematic diagram of the thermal position sensor with a differential amplifier circuit.

\section{DESCRIPTION OF ELECTROTHERMAL BASED NANOPOSITIONER}

The conceptual schematic view of the nanopositioner is presented in Fig. 1. The device is micro-fabricated from single-crystal silicon using a commercial bulk silicon micromachining technology-SOIMUMP in MEMSCAP [36]. This process has a $25 \mu \mathrm{m}$ thick silicon device layer and a minimum feature/gap of $2 \mu \mathrm{m}$. The Scanning Electron Microscope (SEM) image of the whole device and a section of it are provided in Fig. 2. The position sensors are two beamshaped resistive heaters made from the doped silicon. Application of a fixed dc voltage across the heaters results in a current passing through them, thereby heating the beams. As a heat sink, a rectangular plate is placed beside the beam heaters with a $2 \mu \mathrm{m}$ air gap. The positioner stage is actuated by a thermal actuator, as illustrated in Fig. 2.

Before applying a voltage across the actuator, the positioner stage is at the initial rest position (dashed box in Fig. 1), where the two edges of the sink plate are exactly aligned with the middle of the two thermal resistive sensors $R_{1}$ and $R_{2}$. The sensors are biased by a dc voltage source $V_{d c}$, and the heat generated in the resistive heater is conducted through the air to the heat sink plate (positioner stage). As the plate is centered between the two thermal sensors, the heat fluxes out of the sensors are identical, thereby equaling the temperature and resistance of the sensors. After applying a voltage on the actuator beams, the positioner stage is displaced towards left, the heat flux associated with the sensor on the left increases, while that associated with the sensor on the right decreases, resulting in a decrease in the resistance of the left sensor $\left(R_{l}\right)$, and an increase in resistance of the right sensor $\left(R_{2}\right)$. Thus, the displacement information of the positioner stage can be detected by measuring the resistance difference between the two sensors. The differential changes of the resistance result in current variations in the beam resistors, and the currents are converted to an output voltage using the trans-impedance amplifiers and an instrumentation amplifier. To suppress the common-mode noise, the gains of these two trans-impedance amplifiers must be well matched. Employing the differential topology allows the sensor output to be immune from undesirable drift effects due to changes in ambient temperature or aging effects.

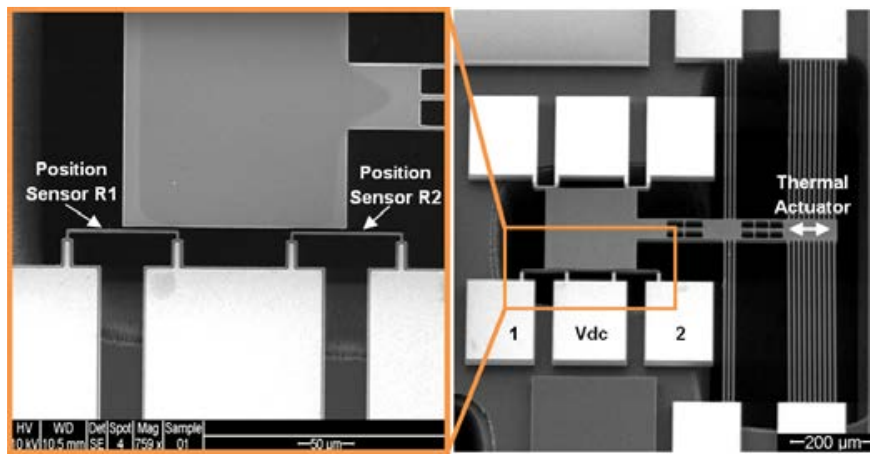

Fig. 2. SEM images of the micromachined nanopositioner.

\section{SENSOR ANALYSIS}

The objective of this section is to predict the sensor temperature and resistance as a function of sensor bias and actuator displacement. To simplify the analysis, lumped parameter approach and constant stationary conditions are considered. This means we assume a uniform temperature distribution in each sensing resistor.

\section{A. Resistivity Temperature Profile}

As the sensing resistors are made from highly doped Silicon, their resistivity is strongly dependent on their temperature. Since the resistivity of un-doped silicon is very high, the resistance of each sensing resistor is almost equal to that of the highly doped layer. Let us assume that the depth of the highly doped layer is $2 \mu \mathrm{m}$ with a uniform donor dopant concentration of $n_{0}=10^{20} \mathrm{~cm}^{-3}$. At room temperature $(300 \mathrm{~K})$, the resistivity model mentioned in [33] predicts an electric resistivity of $1.56 \times 10^{-5} \Omega \mathrm{m}$. Hence, the sensor beam can be considered as a $100 \mu \mathrm{m}$ length bar whose cross section is a square with $2 \mu \mathrm{m}$ sides. For this bar, the calculated resistivity yields a $390 \Omega$ resistance, which agrees with the experimental results (the first row of Table 2). With the selected dopant concentration, the resistivity model in [33] predicts a temperature profile for electrical resistivity $\rho$, as shown in Fig. 3 with the thick curve. From this profile, the differential temperature coefficient of resistivity, defined by:

$$
\alpha(T) \equiv \frac{1}{\rho(T)} \frac{d \rho(T)}{d T}
$$

is obtained in terms of temperature $T$ as shown in Fig. 4. The straight line in Fig. 3 is a rough linear approximation of resistivity for temperatures less than $1500 \mathrm{~K}$, which is described as:

$$
\rho(T) \approx \rho_{\mathrm{amb}}\left[1+\alpha_{\mathrm{o}}\left(T-T_{\mathrm{amb}}\right)\right],
$$

where $\alpha_{0}=3.7822 \times 10^{-4}\left(1 /{ }^{\circ} \mathrm{C}\right)$ is the temperature coefficient of resistivity, $T_{\mathrm{amb}}$ is the ambient temperature, and $\rho_{\mathrm{amb}}$ refer to resistivity at ambient temperature.

\section{B. Linear Analysis}

The results in following example, which is based on lumped parameter analysis for prediction of temperature in terms of 


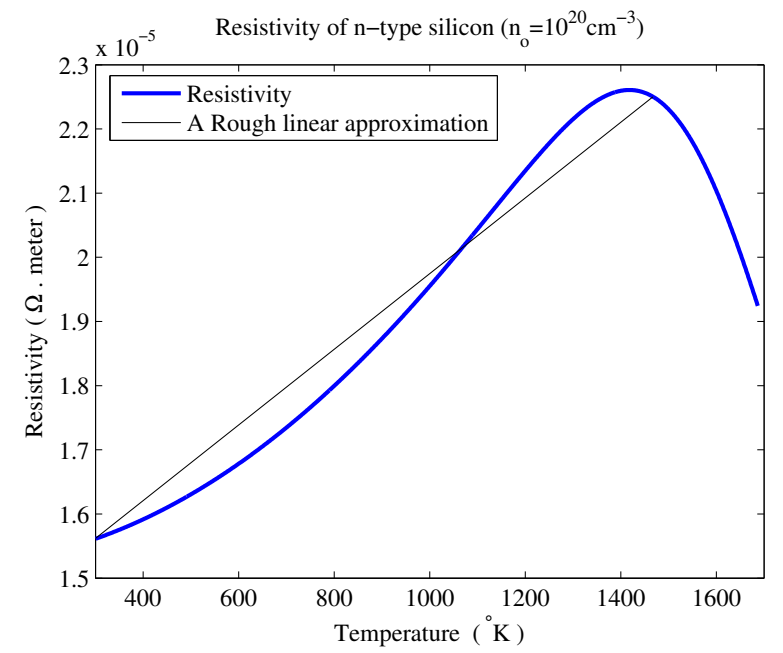

Fig. 3. Temperature profile of resistivity for n-type silicon $\left(n_{0}=10^{20} \mathrm{~cm}^{-3}\right)$.

the applied voltage, will be used subsequently.

Example III-B: Electric resistance of a resistor at room temperature is $R_{o}(\Omega)$ and its variation with temperature can be described by a constant temperature coefficient $\alpha\left(1 /{ }^{\circ} \mathrm{C}\right)$. The overall thermal conductance between the resistor and the outside world, including its connection to a voltage source, is $K\left(\mathrm{~W} /{ }^{\circ} \mathrm{C}\right)$. If the internal resistance of the voltage source is negligible compared to the resistor, what would be the steadystate temperature of resistor for a constant voltage of $V$ (vlots) and room temperature of $T_{o}$ ?

Assuming a uniform temperature distribution $T$ across the resistor, we can equate the electric power generated in the resistor with the thermal power flow from the resistor to the outside world:

$$
\frac{V^{2}}{R_{o}\left[1+\alpha\left(T-T_{o}\right)\right]}=K\left(T-T_{o}\right)
$$

When the constant $\alpha$ does not depend on temperature, the resistance value and its temperature can be formulated in the following form:

$$
\begin{gathered}
R=R_{o} \frac{1+\sqrt{1+\frac{4 \alpha V^{2}}{R_{o} K}}}{2} \\
T=T_{o}+\frac{\sqrt{1+\frac{4 \alpha V^{2}}{R_{o} K}}-1}{2 \alpha}
\end{gathered}
$$

Fig. 5 shows the schematic diagram of the heat sink plate and the sensor, which is described by two resistances. At zero actuation, variable $x$ is equal to $0.5 L$ with $L$ referring to the length of each sensor beam. In this way, lengths $x$ and $L-x$ of the right and left sensor beams are not adjacent to the heat sink, respectively. Let us define $K_{1}$ and $K_{2}$ as the overall thermal conductances from electric resistors $R_{1}$ and $R_{2}$ of the sensor to the area around the sensor, respectively. As the plate in Fig. 5 moves to the left, $K_{1}$ increases while $K_{2}$ decreases. Let us assume a simple dependency between the thermal conductances and the plate position $x$, in the following form:

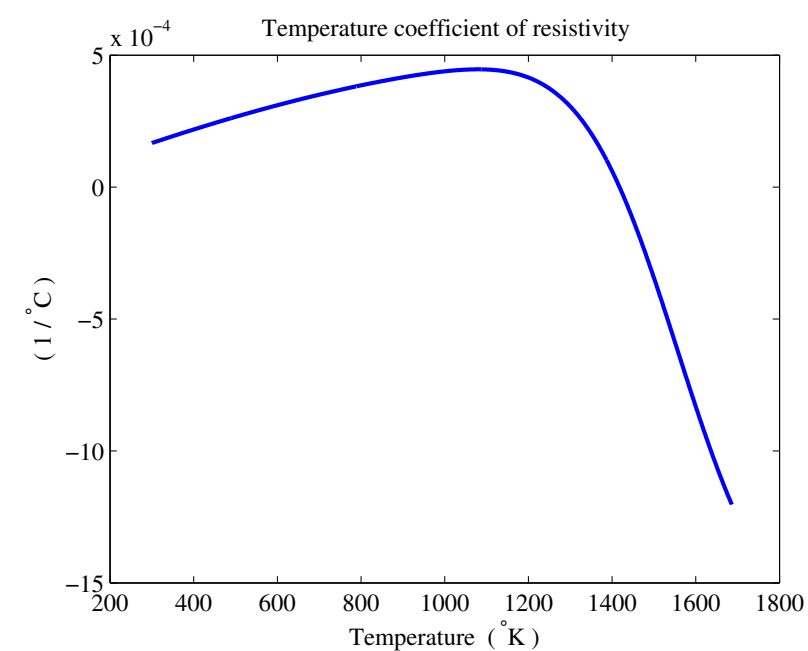

Fig. 4. Temperature profile of the differential temperature coefficient.

$$
\begin{gathered}
K_{1}(\tilde{x})=K_{\text {min }}+\left(K_{\text {max }}-K_{\min }\right) \tilde{x} \\
K_{2}(\tilde{x})=K_{\text {min }}+\left(K_{\text {max }}-K_{\text {min }}\right)(1-\tilde{x})
\end{gathered}
$$

where $\tilde{x} \equiv x / L$ is the normalized position, and $K_{\min }$ and $K_{\max }$ refer to minimum and maximum thermal conductance of each resistor. Neglecting the thermal cross-coupling between the beams, the results in Example III-B can be used to predict the steady-state values of resistances and their corresponding temperatures in the following form:

$$
\begin{aligned}
& R_{i}(\tilde{x}, V)=R_{o} \frac{1+\sqrt{1+\frac{4 \alpha V^{2}}{R_{o} K_{i}(\tilde{x})}}}{2}, i \in\{1,2\} \\
& T_{i}(\tilde{x}, V)=T_{o}+\frac{\sqrt{1+\frac{4 \alpha V^{2}}{R_{o} K_{i}(\tilde{x})}}-1}{2 \alpha}, i \in\{1,2\}
\end{aligned}
$$

where $R_{\mathrm{o}}$ is each resistance value at zero bias voltage. At zero actuation, where $\tilde{x}=0.5$ and $K_{1}=K_{2}=\left(K_{\max }+K_{\min }\right) / 2$, we assume that the sensing resistors match and the sensor output is zero. Hence, the sensor output voltage is proportional to $R_{1}^{-1}-R_{2}^{-1}$. This difference between the electric conductances, which changes with the distance $x$, can be employed to measure the displacement. The following simulation presents the predicted sensor output by the foregoing simple lumped parameter analysis.

Simulation III-B: Assuming the following parameter values and using (6) and (7), the steady-state values of temperatures, resistances, and senor output for different position values are obtained as shown in Fig. 6(a)-(c), respectively.

\section{Thermal Coupling from Actuator to Sensor}

The foregoing simulation predicts an almost linear dependency between the sensor output and displacement. In section III-B, we assumed that the displacement does not have any effect on the temperature of the area around the sensing resistors. In other words, we assumed that the temperature around the sensing resistors, denoted by $T_{\mathrm{o}}$, is identical to the ambient temperature $T_{\mathrm{amb}}$. However, the displacement in the 
TABLE 1

PARAMETER VALUES IN SimULATION III-B.

\begin{tabular}{|l|l|}
\hline Parameter & Value \\
\hline$\alpha$ & $3.7822 \times 10^{-4}\left(\mathrm{~K}^{-1}\right)$ \\
\hline$R_{\mathrm{O}}$ & $390(\Omega)$ \\
\hline$T_{\mathrm{o}}$ & $27\left({ }^{\circ} \mathrm{C}\right)$ \\
\hline$K_{\min }$ & $100 \times 10^{-6}(\mathrm{~W} / \mathrm{K})$ \\
\hline$K_{\max }$ & $116 \times 10^{-6}(\mathrm{~W} / \mathrm{K})$ \\
\hline$V$ & $6(\mathrm{~V})$ \\
\hline
\end{tabular}

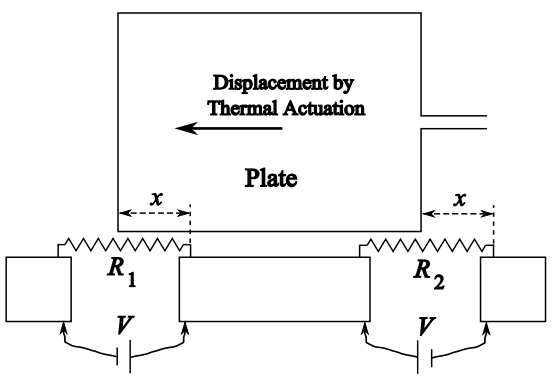

Fig. 5. Biased thermal sensor. (a)

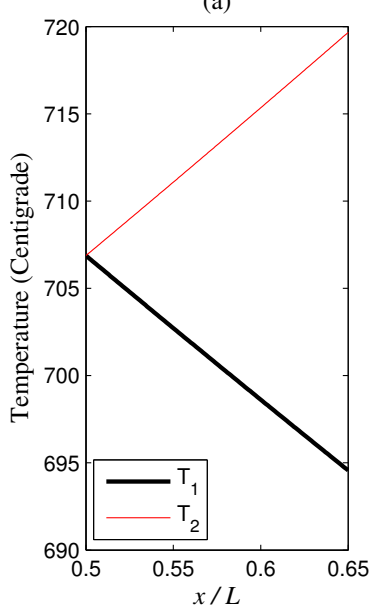

(b)

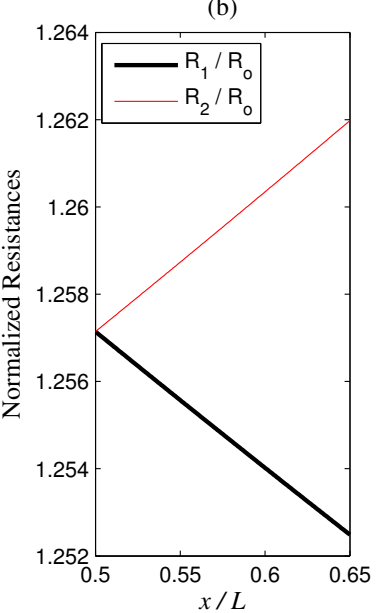

(c)

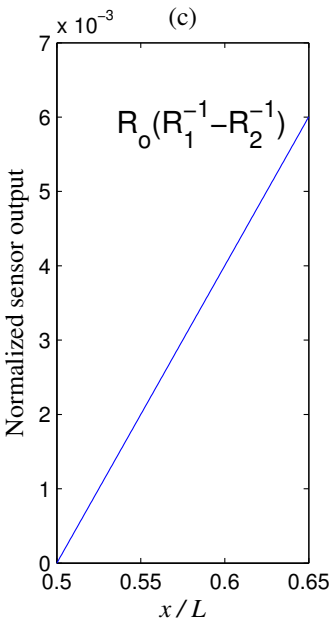

Fig. 6. Steady-state values versus displacement for (a) resistor temperatures, (b) resistor values, and (c) normalized sensor output.

actuator is caused by thermal expansion. Hence, under stationary conditions, the sensor area temperature $\left(T_{\mathrm{o}}\right)$ increases with the displacement $x$. Due to lack of suitable instrumentation to measure the temperature of the sensor area, we set up the following experiment to approximate the sensor area temperature $T_{\mathrm{o}}$.

Experiment III-C (Effect of Actuation on Un-biased Sensor Resistances): For different actuation voltages, the values of sensor resistances were measured, without applying an external bias voltage to the sensor, as shown in Fig. 7 and Table 2. For zero actuation voltage, we can assume that all parts are at room temperature and the values of sensor resistances are almost $390 \Omega$. It is observed that the un-biased sensor resistances increase by about 6.4 percent for a $12 \mu \mathrm{m}$ displacement, just due to transfer of heat from actuator to sensor.

With the temperature coefficient of sensing resistors as given in Table 1 , the 6.4 percent resistance increase in the unbiased sensor corresponds to $169.2{ }^{\circ} \mathrm{C}$ increase in sensor area temperature. Since the edges of the plate align with the middle of sensing resistors at zero actuation, a zero displacement corresponds to $\tilde{x}=0.5$. With $L=100 \mu \mathrm{m}$, the sensor area temperature can be roughly approximated in terms of the normalized displacement and ambient temperature as:

$$
T_{\mathrm{o}} \approx T_{\mathrm{amb}}+1410(\tilde{x}-0.5),\left({ }^{\circ} \mathrm{C}\right)
$$

Using (8) and the given temperature coefficient in Table 1, the value of unbiased sensing resistances in terms of displacement can be written as:

$$
R_{o} \cong R_{\mathrm{amb}}[1+0.53(\tilde{x}-0.5)]
$$

where $R_{\text {amb }}$ refers to sensor resistance at zero actuation and no bias voltage. Now, we can approximate the effect of thermal coupling from the actuator to the biased sensor, in stationary conditions. To do this, we replace $T_{\mathrm{o}}$ and $R_{\mathrm{o}}$ in (7) by their position dependent values on the right hand sides of (8) and (9), respectively. With the same parameters and ambient values as in Simulation III-B, we obtain the results shown in
Fig. 8, where effect of no thermal coupling on sensor output is also included in Fig. 8(c) for comparison.

\section{Effect of Nonlinear Temperature Coefficient}

In the foregoing analysis, we assumed a linear temperature profile for electrical resistivity with a positive temperature coefficient. As shown in Fig. 3, the resistivity is a highly nonlinear function of temperature. Moreover, the differential temperature coefficient, shown in Fig. 4 (up to the melting point of Silicon), becomes negative at high temperatures. If the effect of the nonlinearity is to be taken into account, we can not use closed-from solutions such as Eqs. (4), (5), and (7). Here, we use numerical solutions to consider the effect of the nonlinearity on the sensor under stationary conditions. Let us assume that the resistivity has a known temperature profile, as depicted in Fig. 3. In this way, the heat transfer equation in Example III-B can be written as:

$$
C \frac{d T}{d t}=f(T) \equiv \frac{A V^{2}}{L \rho(T)}-K\left(T-T_{\mathrm{o}}\right)
$$

where $t$ refers to time, $C$ is the heat capacity of the resistor, and $L$ and $A$ are its length and cross sectional area, which are assumed to be constants. For a constant bias voltage $V$, an equilibrium point of $T=T_{\mathrm{e}}$ vanishes the right hand side of Eq. (10), denoted by function $f(T)$. To have an equilibrium point below the melting point $T_{\mathrm{m}}$ of silicon, the minimum value of function $f$ in the interval of $\left[T_{\mathrm{o}}, T_{\mathrm{m}}\right]$ needs to be negative. This condition can be stated as:

$$
\min _{T \in\left[T_{0}, T_{\mathrm{m}}\right]}[f(T)]<0
$$

Because of the nonlinear dependence of $f$ on $T$, we may have more than one solution for the equilibrium. A stationary condition, however, corresponds to a stable equilibrium. Using Eq. (10), the stability of equilibrium can be determined from small temperature perturbation $\delta T \equiv T-T_{\mathrm{e}}$, which satisfies the following linear dynamic equation: 


\begin{tabular}{|c|c|c|c|}
\hline \\
\hline 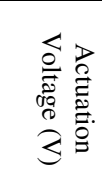 & 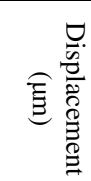 & $\mathrm{R}_{1}(\Omega)$ & $\mathrm{R}_{2}(\Omega)$ \\
\hline 0 & 0 & 388.9 & 391.6 \\
\hline 1 & 0.1 & 389.4 & 391.9 \\
\hline 2 & 0.7 & 391.3 & 394 \\
\hline 3 & 2 & 393.05 & 395.9 \\
\hline 4 & 3.8 & 398 & 400.7 \\
\hline 5 & 5.8 & 401.7 & 404.2 \\
\hline 6 & 8 & 405.5 & 408.1 \\
\hline 7 & 10.3 & 410.2 & 412.5 \\
\hline 8 & 12.8 & 414.8 & 416.8 \\
\hline
\end{tabular}

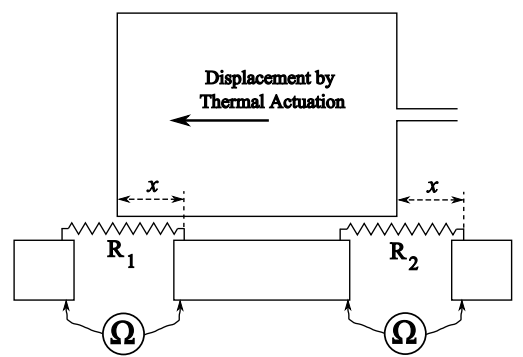

Fig. 7. Measurement of un-biased sensing resistances for different thermal actuation voltages.

$$
C \frac{d \delta T}{d t}=-\left(\frac{A V^{2}}{L \rho^{2}} \frac{d \rho}{d T}+K\right)_{T=T_{\mathrm{e}}} \cdot \delta T
$$

Since the heat capacity is positive, the equilibrium point is stable if the coefficient of $\delta T$ in Eq. (12) is negative. Using Eq. (1), we restrict the solutions to stable equilibrium points by imposing the following condition during the numerical calculations:

$$
\frac{A V^{2} \alpha(T)}{L \rho(T)}+K>0
$$

Here, $\alpha$ has the temperature profile depicted in Fig. 4. To consider the effect of thermal coupling from actuator to sensor, the same approach which led to (8) is adopted. With the nonlinear profile in Fig. 3, a 6.4 percent increase in resistivity corresponds to $263{ }^{\circ} \mathrm{C}$ increase in temperature. Hence, the sensor area temperature is approximated by the following relationship:

$$
T_{\mathrm{o}}(\tilde{x}) \approx T_{\mathrm{amb}}+2192(\tilde{x}-0.5),\left({ }^{\circ} \mathrm{C}\right) .
$$

Dependencies of the thermal conductances $K_{1}$ and $K_{2}$ on position are assumed as stated in Eq. (6). The numerical solutions for resistors' temperatures are obtained from the following equation:

$$
\frac{A V^{2}}{L \rho\left(T_{i}\right)}-K_{i}(\tilde{x})\left[T_{i}-T_{\mathrm{o}}(\tilde{x})\right]=0, \quad i \in\{1,2\}
$$

Having determined the resistor temperatures from (15), the resistance values are obtained as:

$$
R_{i}(\tilde{x})=\frac{\rho\left(T_{i}\right) L}{A}, \quad i \in\{1,2\}
$$

Using the parameter values in Table 3 and restricting the solution to stable equilibriums below the melting point, we obtain the results shown in Fig. 9 under stationary conditions. (b)

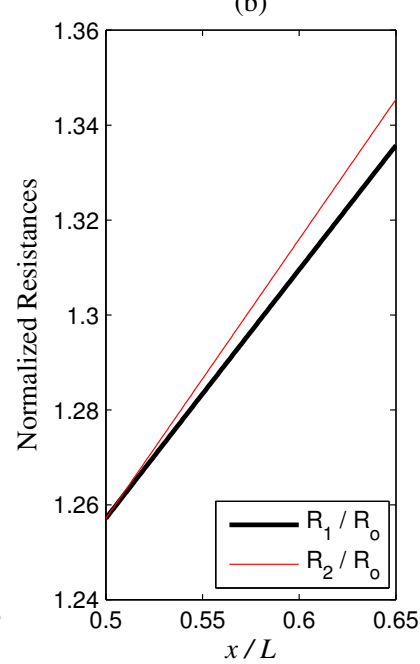

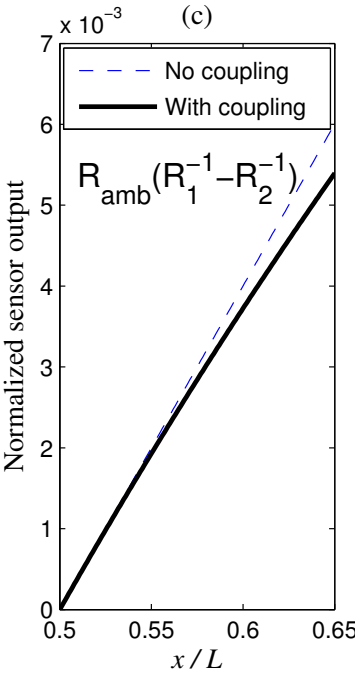

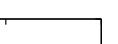


TABLE 3: PARAMETER VALUES.

\begin{tabular}{|l|l|}
\hline Parameter & Value \\
\hline$R_{\mathrm{amb}}$ & $390(\Omega)$ \\
\hline$T_{\mathrm{amb}}$ & $27\left({ }^{\circ} \mathrm{C}\right)$ \\
\hline$T_{\mathrm{m}}$ & $1414\left({ }^{\circ} \mathrm{C}\right)$ \\
\hline$K_{\min }$ & $100 \times 10^{-6}(\mathrm{~W} / \mathrm{K})$ \\
\hline$K_{\max }$ & $116 \times 10^{-6}(\mathrm{~W} / \mathrm{K})$ \\
\hline$V$ & $6(\mathrm{~V})$ \\
\hline
\end{tabular}

(d)

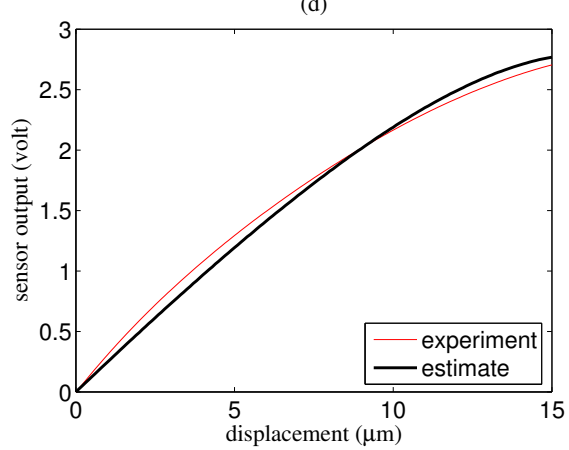

assumption considers a uniform temperature distribution along the beam so that we can use the results of lumped parameter analysis in Example III-B. By the fourth assumption, we assume that the beam remains a straight line after the thermal expansion. In this way, the analysis can be reduced to that of a rigid and linear single beam as shown in Fig. 10.

Using the first assumption and neglecting the voltage drop in the junction area between upper and lower arms, the voltage $V$ across each arm is half of the actuation voltage $V_{\mathrm{a}}$. Assuming a constant thermal conductance $K_{\mathrm{a}}$ between the arm and the surrounding environment and using Eq. (5), the arm temperature in terms of the actuator voltage is obtained as:

$$
T_{\mathrm{a}}=T_{\mathrm{amb}}+\frac{\sqrt{1+\frac{\alpha V_{\mathrm{a}}^{2}}{R_{\mathrm{oa}} K_{\mathrm{a}}}}-1}{2 \alpha}
$$

where $R_{\mathrm{oa}}$ is the electrical resistance of the arm at ambient temperature (no actuation). Having found the arm temperature and assuming a constant linear thermal expansion coefficient for the arm defined by [37]:

$$
\alpha_{l}=\frac{1}{L_{\mathrm{o}}} \frac{d L_{\mathrm{a}}}{d T_{\mathrm{a}}}
$$

where $L_{\mathrm{o}}=\sqrt{d_{\mathrm{o}}^{2}+h^{2}}$ is the arm length at ambient temperature (zero actuation voltage), the arm length $L_{\mathrm{a}}$ is obtained as:

$$
L_{\mathrm{a}}=L_{\mathrm{o}}\left[1+\alpha_{l}\left(T_{\mathrm{a}}-T_{\mathrm{amb}}\right)\right]
$$

Vertical projection of arm, denoted by $h$ in Fig. 10, remains constant after actuation. Hence, the actuator displacement $d$ is obtained in the following form.

$$
d=\sqrt{L_{\mathrm{a}}^{2}-h^{2}}-d_{\mathrm{o}}
$$

where $d_{\mathrm{o}}$ is horizontal projection of the arm at zero actuation.

\section{B. Nonlinear Temperature Profile}

In the linear model, we considered a uniform temperature profile for the beam, which is a very rough approximation. In [38], analytical expressions for temperature profile were (b)
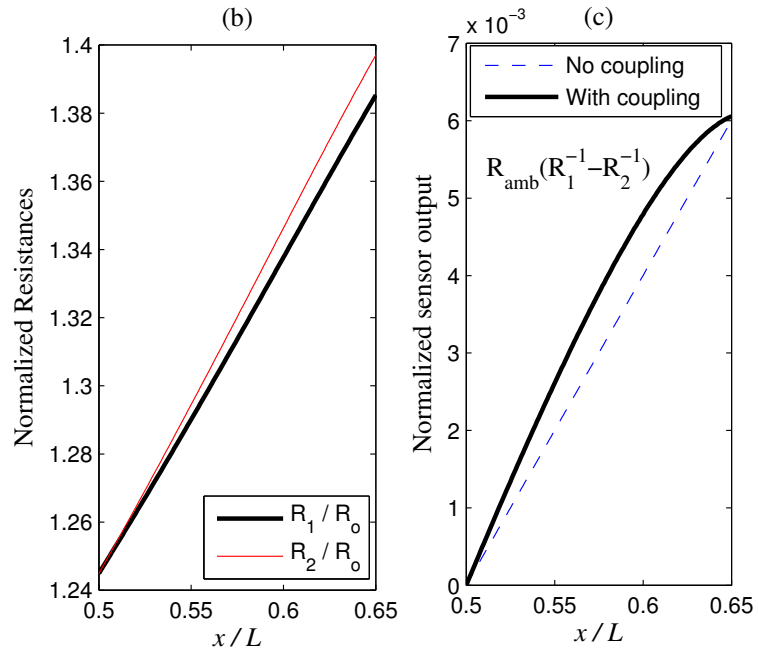

obtained for fixed micromachined polysilicon beams carrying electrical current; however, the effect of thermal expansion was not considered and the equations are only valid for sufficiently high current values. Another analysis was also presented for a bidirectional vertical thermal bimorph actuator in [39]; however, no temperature dependency was considered for the electrical resistivity. Under stationary conditions, temperature profile of the arm can be approximated by the following one dimensional heat equation:

$$
\frac{I^{2}}{A_{\mathrm{e}}} \rho(\tilde{T})+k A \frac{d^{2} \tilde{T}}{d s^{2}}=g_{\mathrm{a}} \tilde{T}
$$

where $\tilde{T}=T(s)-T_{\text {amb }}$ is the temperature increase of the arm at position $s$ on the beam (the s-axis coincides on the beam as shown in Fig. 10), $A_{\mathrm{e}}$ is the effective cross sectional area through which the constant electric current $I$ flows, $k$ is thermal conductivity of the arm, $g_{a}$ refers to thermal conductance per unit length between the arm and ambient region, $A$ is the cross sectional area of the beam, and $\rho$ is the electrical resistivity. To solve the differential equation (20), we assume the following boundary conditions:

$$
\begin{gathered}
G_{L} \tilde{T}\left(L_{\mathrm{a}}\right)+\left.k A \frac{d \tilde{T}}{d s}\right|_{s=L_{\mathrm{a}}}=0 \\
\left.2 k A \frac{d \tilde{T}}{d s}\right|_{s=0}=G_{0} \tilde{T}(0)
\end{gathered}
$$

where $G_{L}$ refers to the overall thermal conductance between the lower end of the arm in Fig. 10 and the ambient region, and $G_{0}$ is the thermal conductance to ambient region for the junction zone between the arm and its upper pair. In (22), a coefficient of 2 appears because two arms are connected to the aforementioned junction zone, where electric heat dissipation is neglected for simplicity.

Let us assume that the electrical resistivity can be approximated by a linear temperature profile described by Eq. (2) and the straight line in Fig. 3. In this case, analytical solutions for the boundary value problem (20-22) are 


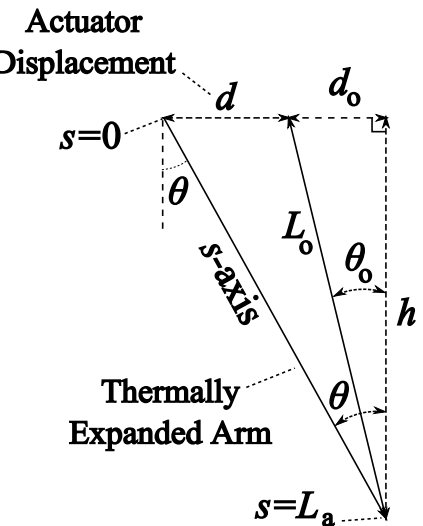

Fig. 10. Schematic of one arm of actuator in the linear model.

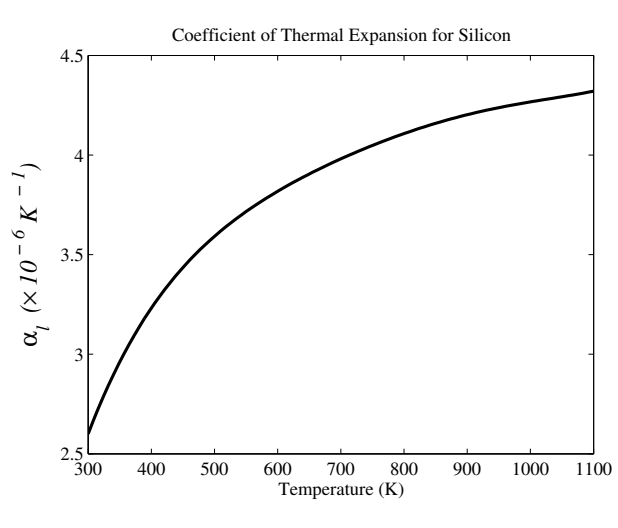

Fig. 11. Schematic Approximate temperature profile of linear thermal expansion coefficient for Silicon.

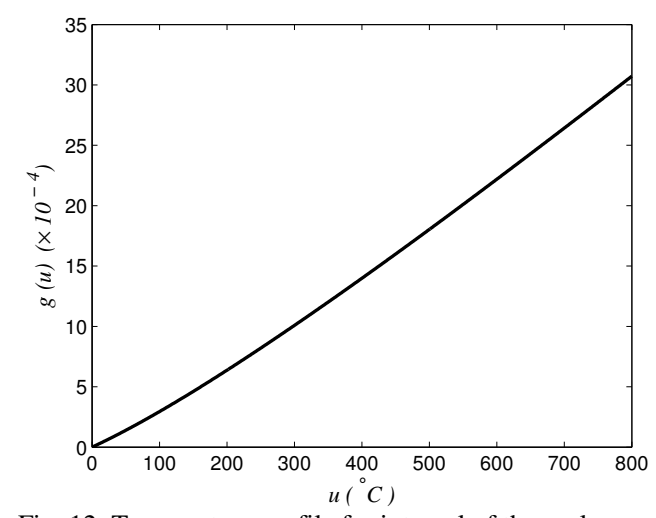

Fig. 12. Temperature profile for integral of thermal expansion coefficient in Fig 11. available. The form of the solution depends on the sign of the following parameter:

$$
\eta:=k^{-1} A^{-1}\left(I^{2} \rho_{\text {amb }} \alpha_{\mathrm{o}} A_{\mathrm{e}}^{-1}-g_{\mathrm{a}}\right)
$$

For positive values of $\eta$, the solution is presented as:

$$
\tilde{T}(s)=C_{1} \sin (\sqrt{\eta} s)+C_{2} \cos (\sqrt{\eta} s)-\frac{\gamma}{\eta}
$$

where

$$
\gamma:=I^{2} \rho_{\mathrm{amb}} k^{-1} A^{-1} A_{\mathrm{e}}^{-1},
$$

and the constants $C_{1}$ and $C_{2}$ satisfy the following linear algebraic equations to meet the boundary conditions:

$$
\begin{gathered}
k A \sqrt{\eta}\left[C_{2} \sin \left(\sqrt{\eta} L_{\mathrm{a}}\right)-C_{1} \cos \left(\sqrt{\eta} L_{\mathrm{a}}\right)\right]= \\
G_{L}\left[C_{1} \sin \left(\sqrt{\eta} L_{\mathrm{a}}\right)+C_{2} \cos \left(\sqrt{\eta} L_{\mathrm{a}}\right)-\eta^{-1} \gamma\right], \\
2 k A \sqrt{\eta} C_{1}=G_{0}\left(C_{2}-\eta^{-1} \gamma\right)
\end{gathered}
$$

For negative values of $\eta$, we can define $\beta:=\sqrt{-\eta}$ and the solution is presented as:

$$
\tilde{T}(s)=C_{1} \sinh (\beta s)+C_{2} \cosh (\beta s)+\frac{\gamma}{\beta^{2}}
$$

where

$$
\begin{gathered}
-k A \beta\left[C_{1} \cosh \left(\beta L_{\mathrm{a}}\right)+C_{2} \sinh \left(\beta L_{\mathrm{a}}\right)\right]= \\
G_{L}\left[C_{1} \sinh \left(\beta L_{\mathrm{a}}\right)+C_{2} \cosh \left(\beta L_{\mathrm{a}}\right)+\beta^{-2} \gamma\right], \\
2 k A \beta C_{1}=G_{0}\left(C_{2}+\beta^{-2} \gamma\right)
\end{gathered}
$$

In the singular case of $\eta=0$, the solution is presented as:

$$
\tilde{T}(s)=C_{2}+C_{1} s-\frac{\gamma}{2} s^{2}
$$

where

$$
\begin{gathered}
k A\left(\gamma L_{\mathrm{a}}-C_{1}\right)=G_{L}\left(L_{\mathrm{a}} C_{1}+C_{2}-\gamma L_{\mathrm{a}}^{2} / 2\right) \\
2 k A C_{1}=G_{0} C_{2}
\end{gathered}
$$

So far, the solution for temperature profile is in terms of the current $I$ and arm length $L_{\mathrm{a}}$. However, the arm length after actuation is not known in advance because it depends on temperature profile of the arm. This dependence can be written analytically if we partition the arm into infinitesimal sections $\Delta s_{i}, i=1, \ldots, N$, which have temperature increases $\tilde{T}_{i}, i=1, \ldots$, $N$, respectively. Assuming a constant thermal expansion coefficient of $\alpha_{l}$ and denoting the un-actuated length of $\Delta s_{i}$ by
$\Delta s_{o i}$, we can write a relationship similar to Eq. (18.b) in the following form:

$$
\Delta s_{o i}=\frac{\Delta s_{i}}{1+\alpha_{l} \tilde{T}_{i}}
$$

When the infinitesimal sections tend to zero while $N$ tends to infinity, a summation over $i=1, \ldots, N$, from both sides of (34) leads to the following constraint on profile solution:

$$
\int_{s=0}^{s=L_{4}} \frac{d s}{1+\alpha_{l} \tilde{T}(s)}=L_{\mathrm{o}}
$$

In this way, after choosing a solution for temperature profile based on sign of $\eta$, the arm length $L_{\mathrm{a}}$ must be adjusted to satisfy constraint (35). The actuator displacement is then obtained from Eq. (19).

\section{Nonlinear Thermal Expansion Coefficient}

In the aforementioned method, we assumed a constant value for coefficient of linear thermal expansion. However, the thermal expansion coefficient for Silicon considerably varies with temperature, as shown in Fig. 11 [37].

To take this variation into account we can use Eq. (18.a) to obtain the modified form of Equation (34) as:

$$
\Delta s_{o i}=\frac{\Delta s_{i}}{1+\int_{0}^{\tilde{T}_{i}} \alpha_{l}(\tilde{T}) d \tilde{T}}
$$

In this way, constraint (35) can be written in the following form:

$$
\int_{s=0}^{s=L_{\mathrm{a}}} \frac{d s}{1+g(\tilde{T}(s))}=L_{\mathrm{o}}
$$

where function $g(\cdot)$ is defined as:

$$
g(u):=\int_{0}^{u} \alpha_{l}(\tilde{T}) d \tilde{T}
$$

For the thermal expansion coefficient profile in Fig. 11, function $g$ has the profile shown in Fig. 12. In this way, after selecting a parameterized form for temperature profile $\tilde{T}\left(s, L_{\mathrm{a}}\right)$ based on sign of $\eta$, the arm length $L_{\mathrm{a}}$ needs to be adjusted to satisfy constraint (37). Having determined the arm length $L_{\mathrm{a}}$, the actuator displacement is obtained from Eq. (19). 


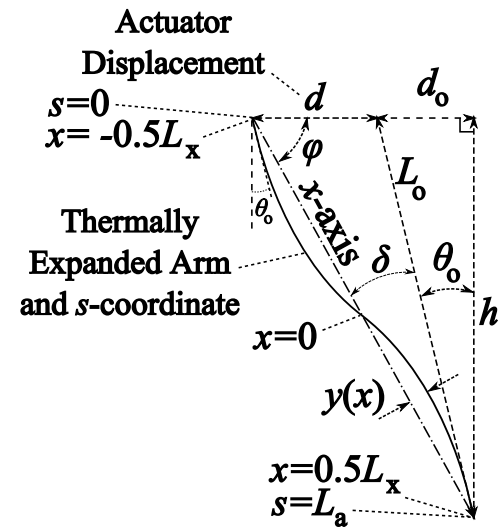

Fig. 13. Nonlinear Profile of Arm.

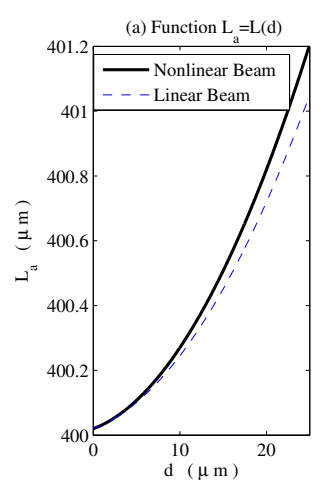

Fig. 14. Profiles of the functions relating arm length $L_{a}$ to displacement $d$ for $h=400$ and $d_{0}=4 \mu \mathrm{m}$.

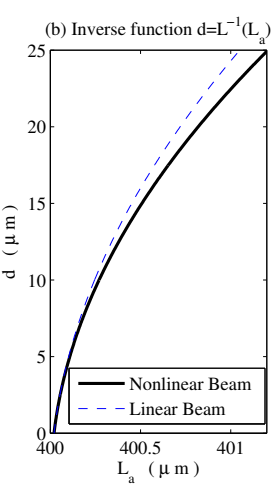

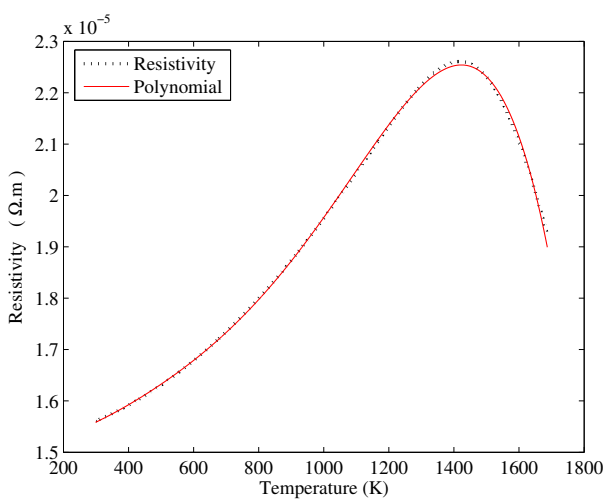

Fig. 15. Approximation of nonlinear resistivity profile by a fifth order polynomial.

\section{Nonlinear Arm Profile (Buckling)}

So far, we have assumed that the shape of the arm remains as a straight line after thermal expansion. However, experiments show that the arm can experience noticeable deviation from straight line in its shape, known as buckling. Buckling in thermal actuators was investigated in [40] by experiment and nonlinear structural finite-element simulations without considering any analytical approaches. Analytical solutions for buckling were also obtained in [41] and [42]; however, the beam conditions in [41] and the buckling source in [42] are different from those considered here. Our analytical approach for buckling is also suitable to include effects of the thermal profile nonlinearities associated with the thermal expansion coefficient and temperature coefficient of resistivity. This problem is mainly due to slender structure of the arm, its clamped connections at the two ends, and fixed vertical projection of arms during thermal expansion (distance $h$ in Fig. 10). Since the end points of the arm are clamped (not hinged), the angle between the arm and vertical direction at the end point junctions, denoted by $\theta$ in Fig. 10, remains constant during thermal expansion (equal to cold value of $\theta_{\mathrm{o}}$ in Fig. 10). Fig. 13 shows a typical shape of the actuated arm, which is more consistent with the foregoing clamped boundary conditions at the beam ends. As shown in Fig. 13, the straight line connecting the beam ends is adopted as $x$-axis, which is used as a reference to measure the arm deflection $y(x)$. Under Euler-Bernoulli beam assumptions and a uniform bending stiffness $E I$, we can write the following differential equation and boundary conditions:

$$
\frac{d^{4} y(x)}{d x^{4}}=0,\left.y\right|_{x= \pm \frac{L_{x}}{2}}=0,\left.\frac{d y}{d x}\right|_{x= \pm \frac{L_{x}}{2}}=\mp \tan \delta
$$

where

$$
\delta(d)=\frac{\pi}{2}-\theta_{\mathrm{o}}-\arctan \left(\frac{h}{d+d_{\mathrm{o}}}\right), L_{\mathrm{x}}(d)=\sqrt{h^{2}+\left(d+d_{\mathrm{o}}\right)^{2}}
$$

In this way, the following shape function is obtained for the thermally expanded arm in terms of the actuator displacement $d$.

$$
y(x)=\left(\frac{x}{2}-\frac{2 x^{3}}{L_{\mathrm{x}}^{2}}\right) \tan \delta
$$

Having determined the shape function of the arm, the arm length $L_{\mathrm{a}}$ can be determined in terms of the actuator displacement as:

$L_{\mathrm{a}}=L(d):=2 \int_{x=0}^{0.5 L_{\mathrm{x}}} \sqrt{1+\left[\frac{d y(x)}{d x}\right]^{2}} d x=2 \int_{x=0}^{0.5 L_{\mathrm{x}}} \sqrt{1+\left(\frac{1}{2}-\frac{6 x^{2}}{L_{\mathrm{x}}^{2}}\right)^{2} \tan ^{2} \delta} d x$

For $h=400 \mu \mathrm{m}$ and $d_{0}=4$, the profiles of function $L_{\mathrm{a}}=L(d)$ and the inverse function $d=L^{-1}\left(L_{\mathrm{a}}\right)$, computed by Eq. (42), are shown in Fig. 14 with solid lines. To compare with the linear beam profile assumption, the corresponding results have also been included in Fig. 14 with dotted lines, using Eq. (19).

In this way, when the $s$-coordinate is adopted to coincide with the curved beam, Equations (20)-(33) and (36)-(38) are still valid for the nonlinear beam. After selecting a parametrized nonlinear temperature profile $\tilde{T}\left(s, L_{\mathrm{a}}\right)$, based on sign of $\eta$, the beam length $L_{\mathrm{a}}$ should be adjusted to satisfy constraint (37), which considers the nonlinearity in thermal expansion coefficient. The actuator displacement is then determined from the inverse function $d=L^{-1}\left(L_{\mathrm{a}}\right)$, which includes the nonlinear beam profile.

\section{E. Nonlinear Resistivity Temperature Profile}

In [43], effect of nonlinear resistivity temperature profile was considered using a numerical finite-element ANSYS model; however, their profile did not include the high temperature part of the profile that has a negative slope. Moreover, their modeling procedure was not clarified by any analytical expressions. However, the numerical method that we offer here is more transparent due to the analytical expressions presented earlier. Up to this point, we assumed a linear temperature profile for electrical resistivity, represented by Eq. (2) and the straight line in Fig. 3. This assumption led to closed-form expressions (23)-(33) for temperature profiles. When the nonlinear dependency of resistivity on temperature, illustrated in Fig. 3, is to be taken into account, the boundary value problem (20)-(22) does not have any closed-form solution and requires a numerical solution. Here, we use a MATLAB solver, "bvp4c", which is suitable for ordinary differential equations with two-point boundary value problems. 
TABLE 4

PARAMETER VALUES USED FOR SIMULATIONS.

\begin{tabular}{|l|l|}
\hline Parameter & Value \\
\hline$d_{\mathrm{o}}$ & $4(\mu \mathrm{m})$ \\
\hline$h$ & $400(\mu \mathrm{m})$ \\
\hline$A$ & $125(\mu \mathrm{m})^{2}$ \\
\hline$A_{\mathrm{e}}$ & $12.5(\mu \mathrm{m})^{2}$ \\
\hline$\alpha_{l}$ (at room temperature) & $2.6 \times 10^{-6}(1 / \mathrm{K})$ \\
\hline$\alpha_{\mathrm{o}}$ & $3.7822 \times 10^{-4}\left(1 /{ }^{\circ} \mathrm{C}\right)$ \\
\hline$k$ & $130\left(\mathrm{WK}^{-1} \mathrm{~m}^{-1}\right)$ \\
\hline$G_{\mathrm{L}}$ & $170 \times 10^{-6}(\mathrm{~W} / \mathrm{K})$ \\
\hline$G_{0}$ & $35 \times 10^{-6}(\mathrm{~W} / \mathrm{K})$ \\
\hline$g_{\mathrm{a}}$ & $0.03\left(\mathrm{WK}^{-1} \mathrm{~m}^{-1}\right)$ \\
\hline$K_{\mathrm{a}}$ & $199.5 \times 10^{-6}(\mathrm{~W} / \mathrm{K})$ \\
\hline$T_{\mathrm{amb}}$ & $300(\mathrm{~K})$ \\
\hline$R_{\mathrm{oa}}$ & $500(\Omega)$ \\
\hline
\end{tabular}

TABLE 5

ELECTRIC CURRENT, ARM LENGTH CHANGE, ARM RESISTANCE, AND ACTUATOR DISPLACEMENT FOR THE PROPOSED MODELS AT ACTUATION

\begin{tabular}{|l|l|l|l|l|}
\hline Nonlinearity type & $\begin{array}{c}I \\
(\mathrm{~mA})\end{array}$ & $\begin{array}{c}L_{\mathrm{a}}-L_{\mathrm{o}} \\
(\mu \mathrm{m})\end{array}$ & $R_{\mathrm{a}}(\Omega)$ & $\begin{array}{c}d \\
(\mu \mathrm{m})\end{array}$ \\
\hline Linear & 8.4 & 0.2 & 536 & 9.17 \\
\hline Temperature Profile & 8.0 & 0.34 & 562 & 13 \\
\hline Thermal Expansion & 8.0 & 0.45 & 562.2 & 15.43 \\
\hline Beam Shape & 8.0 & 0.45 & 562.2 & 14.34 \\
\hline Resistivity & 8.255 & 0.47 & 545 & 14.66 \\
\hline
\end{tabular}

To accelerate the convergence and avoid unacceptable multiple solutions, we used the closed-form solutions, obtained via the linear resistivity assumption, as the initial guess for the solver. Moreover, the nonlinear curve in Fig. 3 is approximated by a fifth order polynomial with adequate precision, as shown in Fig. 15.

\section{F. Simulation Results}

Here we use simulation to evaluate the proposed models for the thermal actuator. The parameter values were consistently selected among different models, as shown in Table 4. Assuming the resistivity profile in Fig. 3, the effective crosssectional area $A_{\mathrm{e}}$ was adjusted to give $R_{\mathrm{oa}}=500 \Omega$ for each arm with length of $400.02 \mu \mathrm{m}$, which is consistent with the measured actuator electrical resistance of $100 \Omega$ at room temperature. For an actuation voltage of $9 \mathrm{~V}$, the temperature profiles of the arm obtained by the proposed models, are shown in Fig. 16. The legends from top to bottom refer to the last type of the nonlinearity included in the model and are orderly associated with the proposed models. In other words, the nonlinearities have not been considered individually, but in a cumulative manner as was explained in the previous sections. Thus, each legend refers to the kind of nonlinearity included in addition to those mentioned in the upper legends. For the simulation results in Fig. 16, the actuator displacement $d$, increase in arm length $L_{\mathrm{a}}-L_{\mathrm{o}}$, the arm electric current $I$, and arm resistance $R_{\mathrm{a}}$ have been tabulated in Table 5. For the nonlinear models, the arm resistance is calculated after obtaining the temperature profile using the following relationship:

$$
R_{\mathrm{a}}=\frac{1}{A_{\mathrm{e}}} \int_{s=0}^{s=L_{\mathrm{a}}} \rho(\tilde{T}(s)) d s
$$

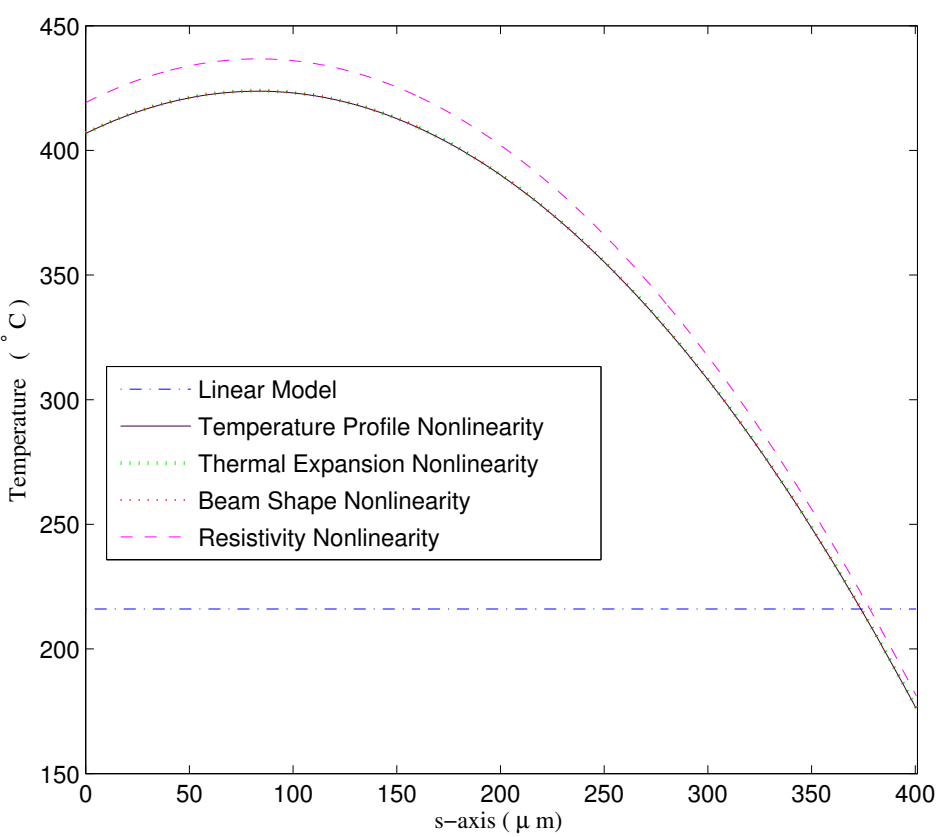

ig. 16. Temperature profiles of the arm with each legend referring to the nonlinearity included in the model in addition to the nonlinearity in the upper legends.

When the thermal expansion nonlinearity is included, the temperature profile is weakly affected in Fig. 16. When the beam shape nonlinearity is added to the temperature profile, length, and resistance of the arm are expectedly not affected (see Table 5). Hence, the profiles associated with the three middle legends almost overlap. However, their displacements have apparent differences as indicated in Table 5. The stationary displacement characteristics of the thermal actuator, obtained by the proposed models, are shown in Fig. 17.

Fig. 18 shows the predicted displacement characteristics, using the model described in Section IV-E that considers all nonlinearities, along with the experimental data. It is seen that the initial deflections predicted by the model are more than the measured values. The reason can be explained by considering the un-excited supporting beams, which were used to transfer the heat outside of the actuator. Evidently, the temperatures of these beams are lower than those of the electrically excited arms and their smaller thermal expansions compared to those of the arms exhibit a resisting force against the motion.

\section{CONCLUSIONS}

Simple lumped parameter methods were used to predict the static behavior of a micro-machined thermal sensor. Effects of thermal coupling from actuator to sensor and nonlinearity of resistivity profile were cumulatively included. The thermal actuation mechanism was first modeled by a simple lumpedparameter model. The model was gradually improved by incorporating more nonlinear effects. The effects, which were cumulatively considered in a step by step manner, are nonuniform temperature profile of the arms, temperature dependency of thermal expansion coefficient, deviation of arm 


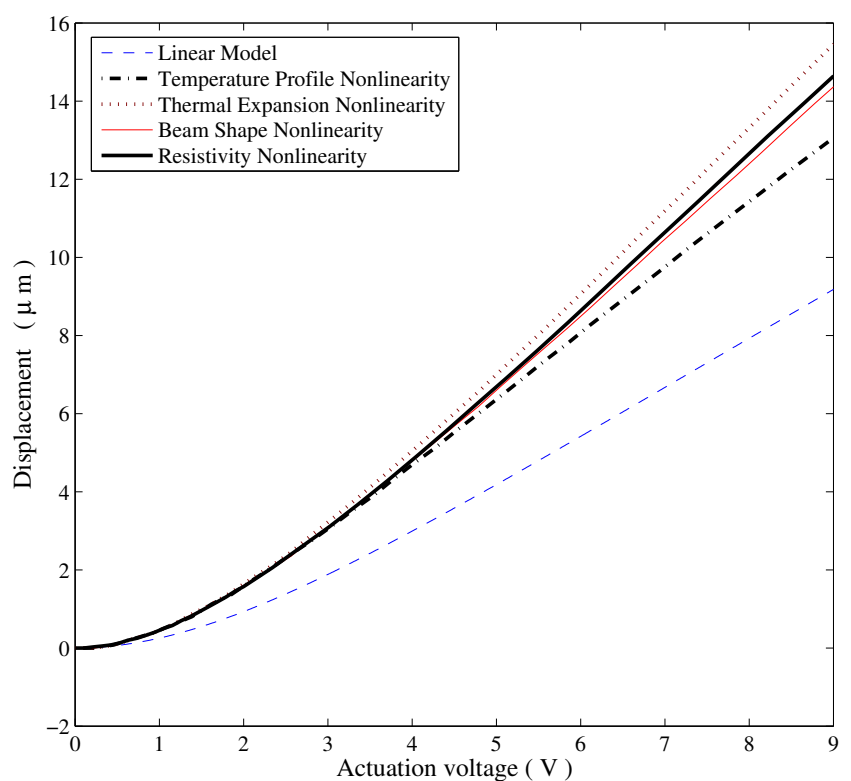

Fig. 17. Stationary displacement characteristics of the actuator predicted by the proposed models.

shape from straight line due to end point constraints, and nonlinearity in resistivity temperature profile. The rough agreement between the resulting sensor and actuator characteristics ensures the validity of the results.

The proposed analytical methods are helpful for predicting sensitivity and power consumption of the thermoelectric sensor and actuator. They also reveal how thermal coupling from actuator to sensor affects the sensor resolution. Moreover, using the proposed methods, one can predict the voltage limitations of the sensor and actuator, which are very important to prevent damages when using high voltage ranges.

\section{REFERENCES}

[1] G. Binnig and H. Rohrer, "Scanning tunneling microscopy," Helvetica Phys. Acta, vol. 55, pp. 726-735, 1982.

[2] G. Binnig, and H. Rohrer, "The scanning tunneling microscope," Sci. Am., vol. 253, pp.50-56, 1986.

[3] G. Binnig, C. Quate, and C. Gerber, "Atomic force microscope," Phys. Rev. Lett., vol. 56, no. 9, pp. 930-933, 1986.

[4] S. Gonda, T. Kurosawa, and Y. Tanimura, "Mechanical performances of a symmetrical, monolithic three-dimensional fine-motion stage for nanometrology,” Meas. Sci. Technol., vol. 10, pp. 986-993, 1999.

[5] T. Ando, N. Kodera, T. Uchihashi, A. Miyagi, R. Nakakita, H. Yamashita, and K. Matada, "High-speed atomic force microscopy for capturing dynamic behavior of protein molecules at work," e-Journal of Surface Science and Nanotechnology, vol. 3, pp. 384-392, 2005.

[6] A. Pantazi, M.A. Lantz, G. Cherubini, and H. Pozidis, and E. Eleftheriou, "A servomechanism for a micro-electro-mechanical-systembased scanning-probe data storage device," Nanotechonology, vol. 15, pp. S612-S621, 2004.

[7] A. Pantazi, A. Sebastian, G. Cherubini, M. Lantz, H. Pozidis, H. Rothuizen, and E. Eleftheriou, "Control of MEMS-based scanningprobe data-storage devices," IEEE Trans. on Nanotechnology, vol. 15, no. 5, pp. 824-841, 2007.

[8] A. Sebastian, A. Pantazi, S. O. R. Moheimani, H. Pozidis, E. Eleftheriou, "Achieving sub nanometer precision in a MEMS storage device during self servo write process," IEEE Transactions on Nanotechnology, vol. 7, no. 5, page 586-595, 2008.

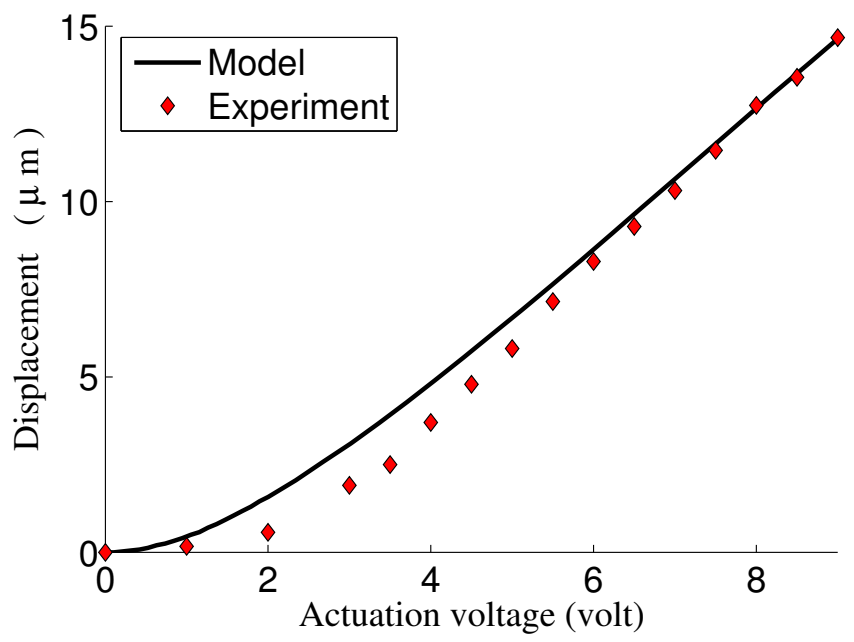

Fig. 18. Experimental and predicted static displacement characteristics of actuator, with the model that includes all four types of nonlinearities.

[9] Y. Sugimoto, P. Pou, O. Custance, P. Jelinek, M. Abe, R. Perez, and S. Morita, "Complex patterning by vertical interchange atom manipulation using atomic force microscopy," Science, vol. 322, pp. 413-417, 2008.

[10] F. Landolsi, F.H. Ghorbel, and J.B. Dabney, "A singular perturbation analysis and control of a new nanomanipulator," 49th IEEE Conference on Decision and Control, pp. 3451-3456, 15-17 Dec. 2010.

[11] G. Binnig and H. Rohrer, "Scanning tunneling microscopy," Physica $B+C$, vol. 127, pp. 37-45, 1984 .

[12] G. Binnig and D.P.E. Smith, "Single-tube three-dimensional scanner for scanning tunneling microscopy," Rev. Sci. Instrum., vol. 57, no. 8, pp. $1688-1689,1986$

[13] A. Bazaei, Y.K. Yong, S.O.R. Moheimani, and A. Sebastian, "Tracking of triangular references using signal transformation for control of a novel AFM scanner stage," IEEE Transactions on Control Systems Technology, ISSN: 1063-6536.

[14] S. Hoen, Q. Bai, J.A. Harley, D.A. Horsley, F. Matta, T. Verhoeven, J. Williams, and K.R. William, "A high-performance dipole surface drive for large travel and force," TRANSDUCERS '03, $12^{\text {th }}$ International Conference on Solid State Sensors, Actuators and Microsystems, pp. 344-347, Boston, June 8-12, 2003.

[15] Y.K. Yong and S.O.R. Moheimani, "A compact XYZ scanner for fast atomic force microscopy in constant force contact mode,", IEEE/ASME International Conference on Advanced Intelligent Mechatronics, Montreal, Canada, pp. 225-230, 6-9 July 2010.

[16] Y.K. Yong, K. Liu, and S.O.R. Moheimani, "Reducing cross-coupling in a compliant XY nanopositioner for fast and accurate raster scanning," IEEE Transactions on Control Systems Technology, vol. 18, no. 5, pp. 1172-1179, 2010.

[17] S. Aphale, S. Devasia, S. O. R. Moheimani, "High bandwidth control of a piezoelectric nanopositioning stage in the presence of plant uncertainties," Nanotechnology, vol. 19, no. 12, 125503, 2008.

[18] Y.K. Yong, S.S. Aphale, and S.O.R.Moheimani, "Design, Identification, and Control of a Flexure-Based XY Stage for Fast Nanoscale Positioning," IEEE Trans. on Nanotechnology, Vol. 8, No. 1, pp. 46-54, 2009.

[19] Y.K. Yong, B. Ahmed, and S.O.R. Moheimani, "Atomic force microscopy with a 12-electrode piezoelectric tube scanner," Review of Scientific Instruments, Vol. 81, No. 3, 033701, 2010.

[20] C.H. Kim, H.M. Jeong, J.U. Jeon, and Y.K. Kim, "Silicon micro XYstage with a large area shuttle and no-etching holes for SPM-based data storage," Journal of Microelectromechanical Systems, vol. 12, no. 4, pp. 470-478, 2003.

[21] C.K. Pang, Y. Lu, J. Chen, H. Zhu, J. Yang, J. Mou, G. Guo, B.M. Chen, and T.H. Lee, "Design, fabrication, sensor fusion, and control of a micro X-Y stage media platform for probe-based storage systems," Mechatronics, vol. 19, no. 7, pp.1158-1168, 2009.

[22] M. Gnerlich, W. Zhang, H. Donahue, A. Voloshin, and S. Tatic-Lucic, "Novel MEMS-based technology for measuring the mechanical properties of a live biological cell," Proceedings of the $\mathrm{XI}^{\text {th }}$ International Congress and Exposition, Orlando, Florida USA, June 2-5, 2008. 
[23] M.J. Sinclair, "A high force low area MEMS thermal actuator," The Seventh Intersociety Conference on Thermal and Thermomechanical Phenomena in Electronic Systems, Las Vegas, NV , USA, pp. 137-132, 2000.

[24] J.-S. Park, L.L. Chu, A.D. Oliver, and Y.B. Gianchandani, "Bent-beam electrothermal actuators-part II: Linear and rotary microengines," Journal of Microelectromechanical Systems, vol. 10, no.2, pp. 255-262, 2001.

[25] J.M. Maloney, D.S. Schreiber, and D.L. DeVoe, "Large-force electrothermal linear micromotors," J. Micromech. Microeng., vol. 14, no. 2, pp. 226-234, 2004.

[26] A. Geisberger, D. Kadylak and M. Ellis, "A silicon electrothermal rotational micro motor measuring one cubic millimeter," J. Micromech. Microeng., vol. 16, no. 10, pp. 1943-1950, 2006.

[27] R.P. Hogervorst1, B. Krijnen, D.M. Brouwer, J.B.C. Engelen, and U. Staufer, "A single-mask thermal displacement sensor in MEMS," Proceedings of the Tenth International Conference of the European Society for Precision Engineering \& Nanotechnology, Delft, The Netherlands. pp. 462-465, 31 May-June 42010.

[28] M.A. Lantz, G.K. Binning, M. Despont, and U. Drechsler, "A micromechanical thermal displacement sensor with nanometre resolution," Nanotechnology, vol. 16, no. 8, pp. 1089-1094, 2005.

[29] G.K. Binning, M. Despont, M.A. Lantz, and P. Vettiger, "Thermal movement sensor," International patent application, WO 2004/020328 A1.

[30] R.K. Messenger, Q.T. Aten, T.W. McLain, and L.L. Howell, "Piezoresistive feedback control of a MEMS thermal actuator," Journal of Microelectromechanical Systems, vol. 18, no.6, pp. 1267-1278, 2009.

[31] Y. Zhu, A. Bazaei, S. O. R. Moheimani, and M. Yuce, A micromachined nanopositioner with on-chip electrothermal actuation and sensing, IEEE Electron Device Letters, Vol. 31, No. 10, pp. 1161$1163,2010$.

[32] N.B. Hubbard, M.L. Culpepper, and L.L. Howell, "Actuators for micropositioners and nanopositioners," Applied Mechanics Reviews, vol. 59, no. 6, pp. 324-334, 2006.

[33] U. Dürig, "Fundamentals of micromechanical thermoelectric sensors," Journal of Applied Physics, vol. 98, no. 4, 044906, 2005.

[34] Y. Zhu, A. Corigliano, and H.D. Espinosa, "A thermal actuator for nanoscale in situ microscopy testing: design and characterization," $J$. Micromech. Microeng., vol. 16, no. 2, pp. 242-253, 2006.

[35] C.D. Lott, T.W. McLain, J.N. Harb, L.L. Howell, "Modeling the thermal behavior of a surface-micromachined linear-displacement thermomechanical microactuator," Sensors and Actuators A, vol. 101, no. 1-2, pp 239-250, 2002.

[36] http://www.memscap.com/en_mumps.html

[37] Y. Okada and Y. Tokumaru, "Precise determination of lattice parameters and thermal expansion coefficient of silicon between 300 and 1500 K", Journal of Applied Physics, vol. 56, no. 2, pp. 314-320, 1984.

[38] L. Lin and M. Chiao, "Electrothermal responses of lineshape microstructures," Sensors and Actuators A: Physical, vol. 55, no. 1, pp. 35-41, 1996.

[39] D. Yan, A. Khajepour, and R. Mansour, "Design and modeling of a MEMS bidirectional vertical thermal actuator," J. Micromech. Microeng., vol. 14, no. 7, pp. 841-850, 2004.

[40] J.W. Wittwer, M.S. Baker, and L.L. Howell, "Simulation, measurement, and asymmetric buckling of thermal microactuators," Sensors and Actuators A: Physical, vol. 128, no. 2, pp. 395-401, 2006.

[41] L. Lin and M. Chiao, "Electro, thermal and elastic characterizations of suspended micro beams," Microelectronics Journal, vol. 29, no. 4-5, pp. 269-276, 1998.

[42] J.W. Wittwer, T. Gomm, and L.L. Howell, "Surface micromachined force gauges: uncertainty and reliability," J. Micromech. Microeng., vol. 12, no. 1, pp. 13-20, 2002.

[43] L.M. Phinney, M.A. Spletzer, M.S. Baker, and J.R. Serrano, "Effects of mechanical stress on thermal microactuator performance," $J$. Micromech. Microeng., vol. 20, no. 9, 095011, 2010. 